\title{
EXCITONIC MOLECULE IN WURTZITE CRYSTALS
}

\author{
W. Ungier, P. JANISZEWSKI AND M. SUFFCZYŃSKI \\ Institute of Physics, Polish Academy of Sciences \\ Al. Lotników 32/46, 02-668 Warszawa, Poland \\ (Received July 23, 1993; in final form November 3, 1993)
}

\begin{abstract}
We calculate with the variational technique the fine structure of a biexciton in wurtzite crystals with the effective electron-hole exchange interaction taken into account. The values of the electron-hole exchange integrals are taken from the free exciton $\Gamma_{5}-\Gamma_{6}$ splitting. We calculate the biexciton dissociation energy and the ratio of mixing of the symmetric and antisymmetric envelopes which arises from the electron-hole exchange interaction. Results are presented for $\mathrm{CdS}, \mathrm{CdSe}$ and $\mathrm{ZnS}$ crystals.
\end{abstract}

PACS numbers: $71.35 .+\mathrm{z}$

\section{Introduction}

The biexciton as the bound state of two Wannier excitons is described in the effective mass approximation. The electron-hole exchange interaction mixes two singlet states of opposite parity, therefore the ground state of the biexciton has no definite parity under separate permutation of the spatial and the spin coordinates of identical particles, two electrons and two holes, respectively. It was shown by Ungier [1,2] and Hayashi [3] in previous papers that in the simple band structure (without total angular momentum classification according to spin-orbit interaction) the ground state of the biexciton should be described by a two component, symmetric and antisymmetric, spatial envelope function, but not only the symmetric one.

The present paper is a continuation of the work about biexcitons in $\mathrm{CuCl}$ crystal [4]. The structure of the valence band Bloch functions at $k=0$ for wurtzite crystals differs from that for $\mathrm{CuCl}$ and the exciton exchange splitting in wurtzite $\mathrm{CdS}$ and $\mathrm{ZnS}$ is much greater than in $\mathrm{CuCl}$. The effect of electron-hole exchange mixing of the singlets of opposite parities in $\mathrm{CdS}$ and $\mathrm{ZnS}$ turn out to be of about $20 \%$, while in $\mathrm{CuCl}$ is of about $5 \%$. 


\section{Biexciton states in two-band model of semiconductor}

Semiconductors CdS, CdSe and $\mathrm{ZnS}$, as crystals of the wurtzite structure, possessing a uniaxial symmetry of group $C_{6 v}$, have a band structure characterized with the top of the valence band at $k=0$ (symmetry $\Gamma_{9}$ ) and the bottom of the conduction band also at $k=0$ (symmetry $\Gamma_{7}$ ). The notation of Koster et al. [5] for the irreducible representations of space groups is used throughout this paper. Two states of the valence band, both of symmetry $\Gamma_{7}$, are split off from the $\Gamma_{9}$ state by the crystal field and the spin-orbit interaction.

The conduction Bloch functions of $\Gamma_{7}$ can be written as

$$
c_{\frac{1}{2}}=\phi_{\mathrm{c}}|\uparrow\rangle \text { and } c_{-\frac{1}{2}}=\phi_{\mathrm{c}}|\downarrow\rangle
$$

and the top valence Bloch functions of $\Gamma_{9}$ are of the form [5]:

$$
v_{\frac{3}{2}}=\psi_{1}|\uparrow\rangle \text { and } v_{-\frac{3}{2}}=\psi_{-1}|\downarrow\rangle \text {, }
$$

where $\psi_{ \pm 1}=(1 / \sqrt{2})\left(p_{x} \pm \mathrm{i} p_{y}\right)$ and $p_{x}, p_{y}$ are the $p$-like functions. The states $|\uparrow\rangle$ and $|\downarrow\rangle$ are the pure spin eigenstates of the spin operator of $s=\frac{1}{2}$. Because of the anisotropy of the wurtzite crystals, the states (1) and (2) are no longer eigenstates of total $J$ but only of $J_{z}$.

We assume that the one-electron Bloch functions

$$
\psi_{n, k}=\frac{1}{\sqrt{N}} \mathrm{e}^{\mathrm{i} k \cdot r} u_{n, k}(r) \quad(n=\mathrm{c}, \mathrm{v}),
$$

where $k$ stands for $k, m_{J} ; u_{n, k}$ is a periodic function with the period of the crystal lattice and is normalized to unity in a unit cell of volume $\Omega$. The $\psi_{n, k}$ are normalized in a large volume $V=N \Omega$ and satisfy the usual Hartree-Fock equation

$$
H_{\mathrm{HF}} \psi_{n, k}=E_{n}(k) \psi_{n, k} .
$$

For the $2 N$-electron problem described by the Hamiltonian

$$
H=\sum_{i}^{2 N}\left\{-\frac{\hbar^{2}}{2 m} \Delta_{i}+V_{\text {per }}\left(r_{i}\right)\right\}+\frac{1}{2} \sum_{i \neq j} \frac{e^{2}}{r_{i j}}
$$

the ground state is given by a Slater determinant

$$
|\phi\rangle_{0}=((2 N) !)^{-1 / 2} \operatorname{det}\left\{\left|\psi_{\mathrm{v}, k_{1}}(1) \psi_{\mathrm{v}, k_{2}}(2) \ldots \psi_{\mathrm{v}, k_{2 N}}(2 N)\right\rangle\right\} .
$$

We will assume the biexciton wave function as a linear combination of the states $\left|\phi_{k k^{\prime}}^{l l^{\prime}}\right\rangle$, being Slater determinants in which two-valence wave functions $\left|\psi_{\mathrm{v}, k}(i)\right\rangle$ and $\left|\psi_{\mathrm{v}, k^{\prime}}\left(i^{\prime}\right)\right\rangle$ are substituted by conduction wave functions $\left|\psi_{\mathrm{c}, l}(i)\right\rangle$ and $\left|\psi_{\mathrm{c}, l^{\prime}}\left(i^{\prime}\right)\right\rangle$ in Eq. (6).

The restriction to the subspace of the $\left|\phi_{k k^{\prime}}^{l l^{\prime}}\right\rangle$ allows the formal reduction of the $2 N$-electron biexciton problem to a few particle problem by associating to each function $\left|\phi_{k k^{\prime}}^{l} l^{l} l^{\prime}\right\rangle$ the two-electron and two-hole function

$$
\begin{aligned}
& \left|\bar{\phi}_{k k^{\prime}}^{l l^{\prime}}\right\rangle=\frac{1}{2}\left[\psi_{\mathrm{c}, l}\left(\boldsymbol{r}_{1}\right) \psi_{\mathrm{c}, l^{\prime}}\left(\boldsymbol{r}_{3}\right)-\psi_{\mathrm{c}, l^{\prime}}\left(\boldsymbol{r}_{1}\right) \psi_{\mathrm{c}, l}\left(\boldsymbol{r}_{3}\right)\right] \\
& \quad \times K(2) K(4)\left[\psi_{\mathrm{v}, k}\left(\boldsymbol{r}_{2}\right) \psi_{\mathrm{v}, k^{\prime}}\left(\boldsymbol{r}_{4}\right)-\psi_{\mathrm{v}, k^{\prime}}\left(\boldsymbol{r}_{2}\right) \psi_{\mathrm{v}, k}\left(\boldsymbol{r}_{4}\right)\right],
\end{aligned}
$$


where $K=-\mathrm{i} \sigma_{y} K_{0}$ is the usual time reversal operator and the $K \psi_{\mathrm{v}, k}$ is the state of the hole. Indexes 1,3 refer to the two electrons, indexes 2,4 - to the two holes.

In the subspace spanned by $\left|\bar{\phi}_{k k^{\prime}}^{l l^{\prime}}\right\rangle$ the $2 N$-electron IIamiltonian $H$ is replaced by $H^{(\text {biex })}$, defined by the equation

$$
\left\langle\phi_{k_{2} k_{4}}^{l_{1} l_{3}}|H| \phi_{k_{2}^{\prime} k_{4}^{\prime}}^{l_{1}^{\prime} l_{3}^{\prime}}\right\rangle=\left\langle\bar{\phi}_{k_{2} k_{4}}^{l_{1} l_{3}}\left|H^{\text {(biex) }}\right| \bar{\phi}_{k_{2}^{\prime} k_{4}^{\prime}}^{l_{1}^{\prime}} l_{3}^{\prime} l_{3}^{\prime}\right.
$$

which should be satisfied for all $l, l^{\prime}, k, k^{\prime}$. It was shown by Forney et al. [6] that $H^{\text {(biex) }}$ can be defined as

$$
\begin{gathered}
H^{\text {(biex })}=E_{0}+\sum_{i=1,3} H_{\mathrm{HF}}(i)-\sum_{j=2,4} K(j) H_{\mathrm{HF}}(j) K^{\dagger}(j) \\
+\frac{e^{2}}{\varepsilon}\left(\frac{1}{r_{13}}+\frac{1}{r_{24}}-\frac{1}{r_{12}}-\frac{1}{r_{14}}-\frac{1}{r_{32}}-\frac{1}{r_{34}}\right) \\
+W(1,2)+W(1,4)+W(3,2)+W(3,4),
\end{gathered}
$$

where $E_{0}$ is the ground-state energy of the electrons filling the valence band, $e^{2} / \varepsilon r_{i j}$ are the screened Coulomb interaction terms with the interparticle distances $r_{i j}$ and the static dielectric constant $\varepsilon$. The effective electron-hole exchange term

$$
W(i, j)=V\left(r_{i}, r_{j}\right) V_{\text {spin }}(i, j)
$$

acting on the functions with separate pure spin part $\psi_{n, k}|s\rangle$ (with $|s\rangle=|\uparrow\rangle$ or $|s\rangle=|\downarrow\rangle)$ reads as a projection operator

$$
V_{\text {spin }}(i, j)=\left(\left|\uparrow_{i}\right\rangle\left|\downarrow_{j}\right\rangle-\left|\downarrow_{i}\right\rangle\left|\uparrow_{j}\right\rangle\right)\left(\left\langle\uparrow_{i}\right|\left\langle\downarrow_{j}\right|-\left\langle\downarrow_{i}\right|\left\langle\uparrow_{j}\right|\right)
$$

and

$$
\begin{aligned}
& \left\langle\psi_{\mathrm{c}, l}\left(r_{i}\right) \psi_{\mathrm{v}, k}\left(r_{j}\right)\left|V\left(r_{i}, \boldsymbol{r}_{j}\right)\right| \psi_{\mathrm{c}, l^{\prime}}\left(\boldsymbol{r}_{i}\right) \psi_{\mathrm{v}, k^{\prime}}\left(r_{j}\right)\right\rangle \\
& =\iint \mathrm{d}_{3} r_{i} \mathrm{~d}_{3} r_{j}\left(\psi_{\mathrm{c}, l}\left(r_{i}\right) \psi_{\mathrm{v}, k}\left(r_{i}\right)\right)^{*} \frac{e^{2}}{r_{i j}} \psi_{c, l^{\prime}}\left(r_{j}\right) \psi_{\mathrm{v}, k^{\prime}}\left(\boldsymbol{r}_{j}\right)
\end{aligned}
$$

The lowest exciton and biexciton states are constructed from properly symmetrized electron functions $\Gamma_{7}$ and hole functions $\Gamma_{9}$. The symmetries of the biexciton states are given by the product of the irreducible representations

$$
\begin{gathered}
\Gamma_{7} \otimes \Gamma_{9}^{*} \otimes \Gamma_{7} \otimes \Gamma_{9}^{*}=\left(\Gamma_{5}+\Gamma_{6}\right) \otimes\left(\Gamma_{5}+\Gamma_{6}\right) \\
=2 \Gamma_{1}+2 \Gamma_{2}+2 \Gamma_{3}+2 \Gamma_{4}+2 \Gamma_{5}+2 \Gamma_{6} .
\end{gathered}
$$

The excitons are formed with symmetry $\Gamma_{5}$ :

and $\Gamma_{6}$,

$$
c_{-\frac{1}{2}} v_{\frac{3}{2}} \text { for } J_{z}=+1 \quad \text { or } \quad c_{\frac{1}{2}} v_{-\frac{3}{2}} \text { for } J_{z}=-1
$$

$$
c_{\frac{1}{2}} v_{\frac{3}{2}} \text { for } J_{z}=+2 \quad \text { or } \quad c_{-\frac{1}{2}} v_{-\frac{3}{2}} \text { for } J_{z}=-2 .
$$

$\Gamma_{6}$ corresponds to a superposition of pure triplet spin states, while $\Gamma_{5}$ contains both triplet and singlet spin states. $\Gamma_{5}$ is shifted by $\Delta_{\text {exch }}^{\text {ex }}$ to higher energy by the electron-hole exchange interaction.

The sixteen basis functions $\left|\Gamma_{i} \mu \nu\right\rangle$ of the four particle space of the biexciton can be chosen as those belonging to the irreducible representations of the group $C_{6 v}[7]$ and possessing definite parities under permutation of electrons $(\mu= \pm 1)$ 
and of holes $(\nu= \pm 1)$. Two states belonging to the $\Gamma_{1}$ representation are of the form

$$
\begin{gathered}
\left|\Gamma_{1} \mu \nu\right\rangle=\frac{1}{2}\left(c_{\frac{1}{2}}(1) c_{-\frac{1}{2}}(3)+\mu c_{-\frac{1}{2}}(1) c_{\frac{1}{2}}(3)\right) \\
\times\left(v_{\frac{3}{2}}(2) v_{-\frac{3}{2}}(4)+\nu v_{-\frac{3}{2}}(2) c_{\frac{3}{2}}(4)\right),
\end{gathered}
$$

where $\mu \nu=--$ or $\mu \nu=++$. In order to ensure the proper symmetry of the wave function of identical fermions each $\left|\Gamma_{i} \mu \nu\right\rangle$ of definite permutational parity has to be multiplied by the spatial function of opposite parity

$$
\begin{gathered}
\left|\bar{\mu} \bar{\nu} \begin{array}{cc}
l_{1} & l_{3} \\
k_{2} k_{4}
\end{array}\right\rangle=\frac{1}{2}\left(\mathrm{e}^{\mathrm{i} l_{1} r_{1}+\mathrm{i} l_{3} r_{3}}+\bar{\mu} \mathrm{e}^{\mathrm{i} l_{3} r_{1}+\mathrm{i} l_{1} r_{3}}\right) \\
\times\left(\mathrm{e}^{-\mathrm{i} k_{2} r_{2}-\mathrm{i} k_{4} r_{4}}+\bar{\nu} e^{-\mathrm{i} k_{4} r_{2}-\mathrm{i} k_{2} r_{4}}\right)
\end{gathered}
$$

where $\bar{\mu}=-\mu$ and $\bar{\nu}=-\nu$. In previous treatments $[5,6]$ it was always assumed that each biexciton eigenstate is proportional to one of the $\left|\Gamma_{i} \mu \nu\right\rangle$ of definite parity under the permutation of the two electrons and two holes. In particular, for the ground state the wave functions were chosen to be proportional to the $\left|\Gamma_{1}--\right\rangle$ state $[6,7]$. However, the two states $\left|\Gamma_{1}--\right\rangle$ and $\left|\Gamma_{1}++\right\rangle$ are mixed by the effective electron-hole exchange interaction, and they are not mixed with any other $\left|\Gamma_{i} \mu \nu\right\rangle$ states. Thus the biexciton ground state wave function $|\bar{\phi}\rangle$, expanded in terms of the four particle states, satisfies the stationary Schrödinger equation

$$
H^{\text {(biex) }}|\bar{\phi}\rangle=E_{(\text {biex })}|\bar{\phi}\rangle
$$

and is a linear combination of the states $\left|\Gamma_{1}--\right\rangle$ and $\left|\Gamma_{1}++\right\rangle$,

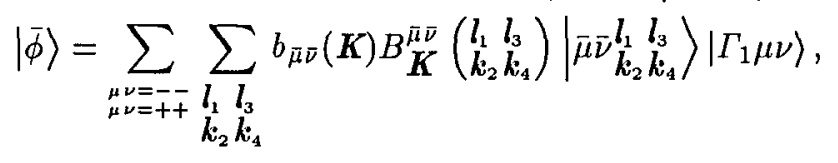

where $\boldsymbol{K}$ denotes the total momentum of the biexciton. The coefficients $B_{\boldsymbol{K}}^{\bar{\mu} \bar{\nu}}\left(\begin{array}{ll}l_{1} & l_{3} \\ k_{2} & k_{4}\end{array}\right)$ are non-vanishing for $l+l^{\prime}-k-k^{\prime}=K$ only.

We assume the normalization condition $\left|b_{++}(\boldsymbol{K})\right|^{2}+\left|b_{--}(\boldsymbol{K})\right|^{2}=1$ and the symmetry relations

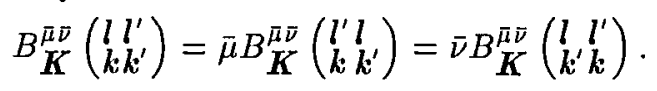

Similarly to the exciton problem we introduce the Fourier transforms [8],

$$
\begin{gathered}
\Phi_{\boldsymbol{K}}^{\bar{\mu} \bar{\nu}}\left(r_{1}, r_{3}, r_{2}, r_{4}\right)=\frac{1}{V^{2}} \sum_{\substack{l_{1} l_{3} \\
k_{2} k_{4}}} B_{K}^{\bar{\mu} \bar{\nu}}\left(\begin{array}{l}
l_{1} l_{3} \\
k_{2} k_{4}
\end{array}\right) \\
\quad \times \exp \left[i\left(l_{1} r_{1}+l_{3} r_{3}-k_{2} r_{2}-k_{4} r_{4}\right)\right]
\end{gathered}
$$

which satisfy the symmetry relations $\Phi_{K}^{\bar{\mu} \bar{\nu}}\left(r_{1}, r_{3}, r_{2}, r_{4}\right)=\bar{\mu} \Phi_{K}^{\bar{\mu} \bar{\nu}}\left(r_{3}, r_{1}, r_{2}, r_{4}\right)=$ $\bar{\nu} \Phi_{\boldsymbol{K}}^{\bar{\mu} \bar{\nu}}\left(\boldsymbol{r}_{1}, \boldsymbol{r}_{3}, \boldsymbol{r}_{4}, \boldsymbol{r}_{2}\right)$. At $\boldsymbol{K}=0$ Eq. (18) can be reduced to the secular equation

$$
\left[\begin{array}{cc}
E_{0}^{++}+\frac{1}{2} \Delta^{++}-E_{(\text {biex })}, & \frac{1}{2} \Delta^{*} \\
\frac{1}{2} \Delta, & E_{0}^{--}+\frac{1}{2} \Delta^{--}-E_{(\text {biex })}
\end{array}\right]\left[\begin{array}{c}
b_{++} \\
b_{--}
\end{array}\right]=0
$$


where

$$
\begin{aligned}
& E_{0}^{\bar{\mu} \bar{\nu}}=\left\langle\Phi^{\bar{\mu} \bar{\nu}}\left|H_{0}\right| \Phi^{\bar{\mu} \bar{\nu}}\right\rangle, \\
& \Delta^{\bar{\mu} \bar{\nu}}=\tilde{J}\left\langle\Phi^{\bar{\mu} \bar{\nu}}\left|\delta_{+}\right| \Phi^{\bar{\mu} \bar{\nu}}\right\rangle, \\
& \Delta=\tilde{J}\left\langle\Phi^{--}\left|\delta_{-}\right| \Phi^{++}\right\rangle,
\end{aligned}
$$

with $\delta_{ \pm}=\Omega\left(\delta\left(r_{1}-r_{2}\right) \pm \delta\left(r_{1}-r_{4}\right)+\delta\left(r_{3}-r_{4}\right) \pm \delta\left(r_{3}-r_{2}\right)\right)$, and

$$
\begin{aligned}
H_{0}= & E_{0}+E_{\mathrm{c}}\left(-\mathrm{i} \nabla_{1}\right)+E_{\mathrm{c}}\left(-\mathrm{i} \nabla_{3}\right)-E_{\mathrm{v}}\left(-\mathrm{i} \nabla_{2}\right)-E_{\mathrm{v}}\left(-\mathrm{i} \nabla_{4}\right) \\
& +\frac{e^{2}}{\varepsilon}\left(\frac{1}{r_{13}}+\frac{1}{r_{24}}-\frac{1}{r_{12}}-\frac{1}{r_{14}}-\frac{1}{r_{32}}-\frac{1}{r_{34}}\right),
\end{aligned}
$$

which represents the kinetic and the Coulomb energy of the four particles. $\tilde{J}$ is the exchange integral for transverse excitons [6,9]. We adopt Dirac $\delta$-functions since the exchange interaction is assumed to be of short-range effective only in the volume $\Omega$ of the unit cell. Equation (22) differs from that for $\mathrm{CuCl}$ by factors in front of electron-hole exchange terms $\Delta[4]$.

\section{The ground state of the biexciton}

In our calculation we take the spherical model of the band energies for the conduction and valence bands in the vicinity of $k=0, E_{\mathrm{c}}(k)=E_{\mathrm{G}}+\hbar^{2} k^{2} / 2 m_{\mathrm{e}}$ and $E_{\mathrm{v}}(k)=-\hbar^{2} k^{2} / 2 m_{\mathrm{h}}$.

To find the ground state of the four-body system we employ a variational technique. We choose for the ground state the lower eigenvalue of Eq. (22)

$$
\begin{aligned}
& E_{(\text {biex })}=\frac{1}{2}\left\{E_{0}^{++}+\frac{1}{2} \Delta^{++}+E_{0}^{--}+\frac{1}{2} \Delta^{--}\right. \\
& \left.-\left[\left(E_{0}^{++}+\frac{1}{2} \Delta^{++}-E_{0}^{--}-\frac{1}{2} \Delta^{--}\right)^{2}+|\Delta|^{2}\right]^{\frac{1}{2}}\right\} .
\end{aligned}
$$

The value of the exchange integral $\tilde{J}$ we take from the measured free exciton $\Gamma_{5}-\Gamma_{6}$ splitting [7]:

$$
\Delta_{\mathrm{exch}}^{\mathrm{ex}}=\tilde{J}|F(0)|^{2} \Omega
$$

with $F(0)$ - the value of the exciton envelope at vanishing electron-hole distance $|F(0)|^{2}=1 / \pi a_{\mathrm{ex}}^{3}$, where $a_{\mathrm{ex}}=\varepsilon \hbar^{2} / \mu e^{2}$ is the exciton Bohr radius with the reduced mass $\mu=m_{\mathrm{e}} /(1+\sigma)$. Here $\sigma=m_{\mathrm{e}} / m_{\mathrm{h}}$ and $a_{\mathrm{ex}}=a_{\mathrm{D}}(1+\sigma)$ where $a_{\mathrm{D}}$ is the neutral donor radius [10]. The energy $E_{(\text {biex })}$, expressed with the parameters of the trial functions $\Phi^{++}$and $\Phi^{--}$, is numerically minimized. The form of $\Phi^{\bar{\mu} \bar{\nu}}$ is that used by Brinkman et al. [11],

$$
\Phi^{\vec{\mu} \bar{\nu}}=[\psi(r) / S(R)] f(R)
$$

where $\psi$ is a function only of the electron-hole distances

$$
\psi=2 \exp \left[-\left(s_{1}+s_{2}\right) / 2\right] \times \begin{cases}\cosh \left[\beta\left(t_{1}-t_{2}\right) / 2\right] & \text { for } \vec{\mu} \vec{\nu}=++ \\ \sinh \left[\beta\left(t_{1}-t_{2}\right) / 2\right] & \text { for } \bar{\mu} \bar{\nu}=--\end{cases}
$$


with $s_{1}=k\left(r_{12}+r_{14}\right), s_{2}=k\left(r_{32}+r_{34}\right), t_{1}=k\left(r_{12}-r_{14}\right), t_{2}=k\left(r_{32}-r_{34}\right)$ and $S^{2}(R)=\int \psi^{2}(r) \mathrm{d}^{3} r_{1} \mathrm{~d}^{3} r_{3}$, while

$$
f(R)=\left[(R / A)^{n} \exp (-R / A)+C \exp (-B R / A)\right] \times \begin{cases}1 & \text { for } \bar{\mu} \bar{\nu}=++, \\ R & \text { for } \bar{\mu} \bar{\nu}=--\end{cases}
$$

with $R$ - the hole-hole separation. The variational parameters $n$ (integer), $k, \beta$, $A, B, C$ of the functions $\Phi^{++}$and the corresponding parameters of $\Phi^{--}$are varied independently in the minimization procedure.

The binding energy of an biexciton is defined as

$$
E_{\mathrm{B}}=2 E_{\mathrm{G}}+E_{0}-E_{(\text {biex })}-2 R_{\mathrm{ex}} \text {, }
$$

where $E_{\mathrm{G}}$ is the gap energy, $E_{0}$ is the ground-state energy of the electrons filling the valence band and $R_{\mathrm{ex}}$ is the free exciton rydberg, $R_{\mathrm{ex}}=\mu e^{4} / 2 \varepsilon^{2} \hbar^{2}$. The calculated binding energies, $E_{\mathrm{B}}$, as well as the ratios of linear coefficients $b_{--} / b_{++}$, which are a measure of the admixture of the antisymmetric envelope function to the symmetric one, are listed in Table.

TABLE

Calculated biexciton energies $E_{\mathrm{B}}$. Input data, taken from [11], are: $\sigma$,

\begin{tabular}{|c|c|c|c|}
\hline & $\mathrm{CdS}$ & $\mathrm{CdSe}$ & $\mathrm{ZnS}$ \\
\hline$\sigma$ & 0.21 & 0.13 & 0.175 \\
\hline$a_{\mathrm{ex}}$ & $29 \AA$ & $47 \AA$ & $21 \AA$ \\
\hline$R_{\mathrm{ex}}$ & 27 & 15 & 36 \\
\hline$\Delta_{\text {exch }}^{\mathrm{ex}}$ & 1.3 & 0.2 & 2.0 \\
\hline $\begin{array}{l}E_{\mathrm{B}} \text { calculated [11] with symmetric envelope } \\
\text { without exchange correction }\end{array}$ & 2.0 & 1.5 & 2.8 \\
\hline $\begin{array}{l}E_{\mathrm{B}} \text { calculated with symmetric envelope } \\
\text { with exchange correction }\end{array}$ & $0.6[6]$ & 1.1 & 0.8 \\
\hline$E_{\mathrm{B}}$ present results & 0.8 & 1.2 & 1.2 \\
\hline$b_{--} / b_{++}$ & 0.17 & 0.05 & 0.20 \\
\hline
\end{tabular}
$a_{\mathrm{ex}}, R_{\mathrm{ex}}$ and $\Delta_{\mathrm{exch}}^{\mathrm{ex}}$. All energies are in $\mathrm{meV}$.

\section{Numerical results and discussion}

Biexciton complexes are mainly observed in semiconductors with indirect band gaps. For direct gap semiconductors, and especially for those with wurtzite structure, there is much less experimental data. It has been shown experimentally that the biexciton binding energy in $\mathrm{CdS}$ is equal to $4.8 \mathrm{meV}$ [12]. Here we have calculated this value to be $0.8 \mathrm{meV}$. The agreement between the measured and calculated values is not satisfactory, yet it is better than that $0.6 \mathrm{meV}$ found by Forney et al. [6]. The optimized wave function, Eq. (27), of the biexciton used 
here is unsatisfactory since it cannot reproduce more than about one half of the biexciton binding energy, as is known from the work of Maksimovic et al. [13]. But Ref. [13] does not give the wave function necessary for calculation of the expectation values in formula (25).

For typical semiconductors of wurtzite structure the values of the exchange integral $\tilde{J}$, as well as the volumes of the unit cells are of the same order of magnitude [14]. The squared exciton envelope at vanishing electron-hole distance $|F(0)|^{2}$ depends on the cube of exciton radius. Thus, when the exciton radius $a_{\mathrm{ex}}$ is small, the exchange splitting $\Delta_{\text {exch }}^{\mathrm{ex}}$, Eq. (26), is large and the electron-hole exchange interaction included in the calculations reduces $E_{\mathrm{B}}$ significantly. For large $\Delta_{\text {exch }}^{\text {ex }}$ the mixing of symmetric and antisymmetric envelope components, the measure of which is $b_{--} / b_{++}$, becomes significant and binding energy of biexciton is a little stronger than $E_{\mathrm{B}}$ calculated with exchange correction but with only symmetric envelope function $[6,7]$. Since $b_{--}$is linear coefficient in the envelope it can be important, for large $\Delta_{\mathrm{exch}}^{\mathrm{ex}}$, in calculations of biexciton-exciton transitions.

\section{References}

[1] W. Ungier, Acta Phys. Pol. A 73, 337 (1988).

[2] W. Ungier, Solid State Commun. 69, 53 (1989).

[3] H. Hayashi, Phys. Rev. B 38, 4016 (1988).

[4] W. Ungier, Acta Phys. Pol. A 83, 703 (1993).

[5] K.F. Koster, J.O. Dimmock, R.G. Wheeler, H. Statz, Properties of the Thirty-Two Point Groups, M.I.T. Press, Cambridge, Massachusetts 1963.

[6] J.J. Forney, A. Quattropani, F. Bassani, Nuovo Cimento B 22, 153 (1974).

[7] F. Bassani, J.J. Forney, A. Quattropani, Phys. Status Solidi B 65, 591 (1974).

[8] J.O. Dimmock, in: Semiconductors and Semimetals, Vol. III, Eds. R.K. Willardson, A.C. Beer, Academic Press, New York 1967, p. 259.

[9] Y. Onodera, Y. Toyozawa, J. Phys. Soc. Jpn. 22, 833 (1967).

[10] G. Staszewska, M. Suffczyński, W. Ungier, L. Wolniewicz, J. Phys. C 17, 5171 (1984).

[11] W.F. Brinkman, T.M. Rice, B. Bell, Phys. Rev. B 8, 1570 (1973).

[12] B. Honerlage, R. Levy, J.B. Grun, C. Klingshirn, K. Bohnert, Phys. Rep. 124, 161 (1985).

[13] G. Maksimovic, I.V. Puzynin, T.A. Strich, F.R. Vukajlovic, Phys. Rev. B 38, 3351 (1988).

[14] M. Suffczyński, L. Świerkowski, W. Wardzyński, J. Phys. C 8, L25 (1975). 